\title{
Systematic studies of the microbunching instability at very low bunch charges
}

\author{
Miriam Brosi, Johannes L. Steinmann, Edmund Blomley, Tobias Boltz, Erik Bründermann, \\ Julian Gethmann, Benjamin Kehrer, Yves-Laurent Mathis, Alexander Papash, Manuel Schedler, ${ }^{\dagger}$ \\ Patrik Schönfeldt, ${ }^{*}$ Patrick Schreiber, Marcel Schuh, \\ Markus Schwarz, ${ }^{\S}$ and Anke-Susanne Müller \\ Laboratory for Applications of Synchrotron Radiation, Karlsruhe Institute of Technology, \\ Kaiserstraße 12, D-76131, Germany \\ Institute for Beam Physics and Technology, Karlsruhe Institute of Technology, \\ Hermann-von-Helmholtz-Platz 1, D-76344 Eggenstein-Leopoldshafen, Germany \\ Michele Caselle, Lorenzo Rota, and Marc Weber \\ Institute for Data Processing and Electronics, Karlsruhe Institute of Technology, \\ Hermann-von-Helmholtz-Platz 1, D-76344 Eggenstein-Leopoldshafen, Germany \\ Peter Kuske \\ Humboldt-Innovation GmbH and Helmholtz-Zentrum Berlin, Hahn-Meitner-Platz 1, \\ D-14109 Berlin, Germany
}

(Received 3 July 2018; published 13 February 2019)

\begin{abstract}
At KARA, the Karlsruhe Research Accelerator of the Karlsruhe Institute of Technology synchrotron, the so-called short-bunch operation mode allows the reduction of the bunch length down to a few picoseconds. The microbunching instability resulting from the high degree of longitudinal compression leads to fluctuations in the emitted terahertz radiation, referred to as bursting. For extremely compressed bunches at KARA, bursting occurs not only in one but in two different bunch-current ranges that are separated by a stable region. This work presents measurements of the thresholds and bursting frequencies in both regimes. Good agreement is found between the data and numerical solutions of the Vlasov-Fokker-Planck equation.
\end{abstract}

DOI: $10.1103 /$ PhysRevAccelBeams.22.020701

\section{INTRODUCTION}

The Karlsruhe Research Accelerator (KARA) is the storage ring of the accelerator test facility and synchrotron light source of the Karlsruhe Institute of Technology (KIT) in Germany. A special short-bunch operation mode at $1.3 \mathrm{GeV}$ allows the reduction of the momentum compaction factor and therefore reduces the electron bunch length down to a few picoseconds. A bunch-by-bunch feedback system enables custom filling patterns from a single bunch to complex multibunch patterns.

Coherent synchrotron radiation (CSR) is emitted for wavelengths in the order of or longer than the emitting

\footnotetext{
*miriam.brosi@kit.edu

†resent address: Varian PT, Troisdorf, Germany.

${ }^{\ddagger}$ Present address: DLR-VE, Oldenburg, Germany.

${ }^{\S}$ Present address: CERN, Geneva, Switzerland.
}

Published by the American Physical Society under the terms of the Creative Commons Attribution 4.0 International license. Further distribution of this work must maintain attribution to the author(s) and the published article's title, journal citation, and DOI. structure. In the short-bunch operation mode, the effect of CSR plays an important role in the beam dynamics. Stable bunches, below the instability threshold and with a bunch length of a few picoseconds, emit time-independent CSR in the low terahertz $(\mathrm{THz})$ frequency range. The range of $\mathrm{THz}$ frequencies actually depends on the shape of the bunch. As soon as the bunch becomes longitudinally unstable, the temporal variation of the particle distribution in the longitudinal phase space leads to a time-dependent modulation of the emitted CSR. This longitudinal instability is driven by CSR self-interaction (described by the CSR impedance) and is referred to as microbunching [1], due to the formation of substructures on the longitudinal bunch profile. The timevarying substructures lead to strong fluctuations of the emitted power in the $\mathrm{THz}$ range, the so-called bursting. The first observation of an instability, attributed to the CSR impedance, was reported at the Advanced Light Source (ALS) [2]. The minimum bunch current at which this phenomenon occurs is called the bursting threshold. It depends strongly on the natural bunch length and therefore on various machine parameters [3].

At the KIT storage ring KARA [4,5] as well as at the Metrology Light Source (MLS) [6], for very short bunches, 
bursting was additionally observed in a region below the main bursting threshold. Indications also can be seen in measurements at Diamond (see [7], Fig. 6), though it was not discussed further. This additional region of instability was already predicted by simulations in Ref. [3]. In the following, this instability is referred to as short-bunchlength bursting (SBB). In this contribution, we discuss measurements of the SBB instability at KARA and compare them to existing theoretical predictions and numerical simulations.

The paper is structured as follows: After the introduction, theoretical predictions based on a very simple model are reviewed in Sec. II. This is followed by a detailed description of the performed measurements. The results at KARA are compared to existing predictions, available in the literature, in Sec. III. In Sec. IV, new simulations for the actual parameters and machine settings, used during the measurements, are presented. Finally, in Sec. V, these simulation results are compared to the experimental ones, and some first ideas and possibilities are discussed in order to explain the small discrepancies and improve the already good agreement between the experiment and theory.

\section{THEORETICAL DESCRIPTION}

The interaction of the electrons inside an electron bunch with their CSR radiation field, which causes the microbunching instability, can be described by the VlasovFokker-Planck (VFP) equation [1]. The solution depends on how the effects of the conductive beam pipe as boundary conditions for the emitted electromagnetic field are taken into account in the Hamiltonian of the VFP equation. In this contribution, the measurements will be compared to a model which considers the CSR wakefields generated by an electron moving on a purely circular orbit of radius $R$ in between two (perfectly conducting and infinitely long) parallel plates separated by a distance of $2 h$. The singleelectron picture is used to derive the CSR wakefield, which is then convolved with the bunch shape to obtain the wake potential of the entire bunch. Straight sections, where no synchrotron radiation is emitted, are not taken into account. The cross section of the vacuum chamber at KARA changes slightly along the ring, e.g., in insertion devices, but in the dipoles, where the interaction between the radiation and the electrons is considered to take place, the cross section is constant (octagonal with height $32 \mathrm{~mm}$ and width $72 \mathrm{~mm}$ ). With this aspect ratio, the "parallel plates" model appears to be a good choice [8,9].

The resulting dependence for the main threshold of the instability using this parallel plates model was published in 2010 by Bane, Cai, and Stupakov [3]. Using the dimensionless quantities $S_{\mathrm{CSR}}$ and $\Pi$ to parametrize CSR strength and shielding, the main threshold can be described by the simple linear scaling law [3]

$$
\left(S_{\mathrm{CSR}}\right)_{\mathrm{th}}=0.5+0.12 \Pi
$$

$$
\begin{gathered}
\text { with } \Pi=\frac{\sigma_{\mathrm{z}, 0} R^{1 / 2}}{h^{3 / 2}} \\
\text { and } S_{\mathrm{CSR}}=\frac{I_{\mathrm{n}} R^{1 / 3}}{\sigma_{\mathrm{z}, 0}^{4 / 3}} \text {. }
\end{gathered}
$$

Here, $\sigma_{\mathrm{z}, 0}$ is the natural bunch length, $R$ the bending radius, $h$ half of the spacing between the parallel plates, and $I_{\mathrm{n}}$ the normalized bunch current:

$$
I_{\mathrm{n}}=\frac{r_{\mathrm{e}} N_{\mathrm{b}}}{2 \pi \nu_{\mathrm{s}, 0} \gamma \sigma_{\delta, 0}}=\frac{I_{\mathrm{b}} \sigma_{\mathrm{z}, 0}}{\gamma \alpha_{\mathrm{c}} \sigma_{\delta, 0}^{2} I_{\mathrm{A}}}
$$

with $N_{\mathrm{b}}$ the number of electrons, $I_{\mathrm{b}}$ the bunch current, $r_{\mathrm{e}}$ the classical electron radius, $\nu_{\mathrm{s}, 0}$ the nominal synchrotron tune, $\sigma_{\delta, 0}$ the natural energy spread, $\alpha_{\mathrm{c}}$ the momentum compaction factor, $\gamma$ the Lorentz factor, and $I_{\mathrm{A}}$ the Alfvén current. ${ }^{1}$ The natural bunch length is given by [10]

$$
\sigma_{\mathrm{z}, 0}=\frac{\alpha_{\mathrm{c}} c \sigma_{\delta, 0}}{2 \pi f_{\mathrm{s}}} \quad \text { with } \quad f_{\mathrm{s}}=\sqrt{\frac{\alpha_{\mathrm{c}} f_{\mathrm{rf}} f_{\mathrm{rev}} \sqrt{e^{2} V_{\mathrm{rf}}^{2}-U_{0}^{2}}}{E 2 \pi}}
$$

with the synchrotron frequency $f_{\mathrm{s}}$, the beam energy $E$, the $\mathrm{rf}$ frequency $f_{\mathrm{rf}}$, the revolution frequency $f_{\text {rev }}$, the $\mathrm{rf}$ peak voltage $V_{\text {rf }}$, and the radiated energy per particle and revolution $U_{0}$.

Equation (1) was obtained from the results of a VFP solver, based on an algorithm devised by Warnock and Ellison [11]. It is the result of a linear fit to the simulated thresholds. This linear scaling law fits best for high values of $\Pi(\Pi>3)$; however, at lower values the simulated thresholds are significantly higher than the fit. Interestingly, the measured thresholds are described more accurately by the fit (see [12]) than the thresholds obtained by the VFP simulations for these low values of $\Pi$. This is the case for the VFP simulations in Ref. [3] as well as for the ones presented in the following.

The fit, leading to Eq. (1), ignores a dip around $\Pi \approx 0.7$, where the calculated thresholds deviate from the simple linear scaling law [3]. A closer look [3] at the calculated energy spread at this dip reveals a second unstable region in the bunch current, with a threshold below the one expected from the linear scaling law. For the values of $\Pi$ accessible at KARA, it is separated by a stable region from the main instability starting at the threshold described by Eq. (1). This new region corresponds to the short-bunch-length bursting studied in the following. The upper and lower limits of this additional region of instability are predicted to depend not only on the bunch length and thus the shielding parameter, but also on $\beta=1 /\left(2 \pi f_{\mathrm{s}} \tau_{\mathrm{d}}\right)$, which relates the synchrotron frequency $f_{\mathrm{s}}$ and the longitudinal damping time $\tau_{\mathrm{d}}[3,13]$. It was therefore termed a weak instability.

\footnotetext{
${ }^{1}$ Alfvén current $I_{\mathrm{A}}=4 \pi \varepsilon_{0} m_{\mathrm{e}} c^{3} / e=17045 \mathrm{~A}$.
} 


\section{MEASUREMENTS}

\section{A. Measurement principle}

The measurements presented in this paper were obtained with a broadband quasioptical Schottky barrier diode from ACST [14] sensitive in the spectral range from several $10 \mathrm{GHz}$ up to $2 \mathrm{THz}$ with the peak sensitivity around $80 \mathrm{GHz}$, which is operated at room temperature. The THz radiation was detected at the Infrared2 beam line, which provides synchrotron radiation from the entry edge of a dipole magnet [15].

To detect the fluctuations in the emitted $\mathrm{THz}$ radiation for each bunch in a multibunch filling pattern individually, the fast detector was combined with the ultrafast data acquisition (DAQ) system KAPTURE (Karlsruhe pulse taking and ultrafast readout electronics) [16]. The KAPTURE system samples the detector response to the $\mathrm{THz}$ pulse of each bunch at four points [17]. In principle, KAPTURE can sample the signal continuously with the rate of the rf frequency of KARA $(\approx 500 \mathrm{MHz})$. For this publication, the signal was recorded for every bunch at every tenth revolution during a period of one second, to limit the acquired data volume.

Figure 1 shows the characteristic patterns of the fluctuation frequencies of the emitted $\mathrm{THz}$ radiation for different bunch currents. During the decay of the bunch current, the instability passes different regimes and ends at the main bursting threshold (in Fig. 1, at $\approx 0.2 \mathrm{~mA}$ ).

The combination of a custom filling pattern (tailored with a bunch-by-bunch feedback system [18]) and a data acquisition system which facilitates the measurement of the $\mathrm{THz}$ signal of each bunch individually allows a

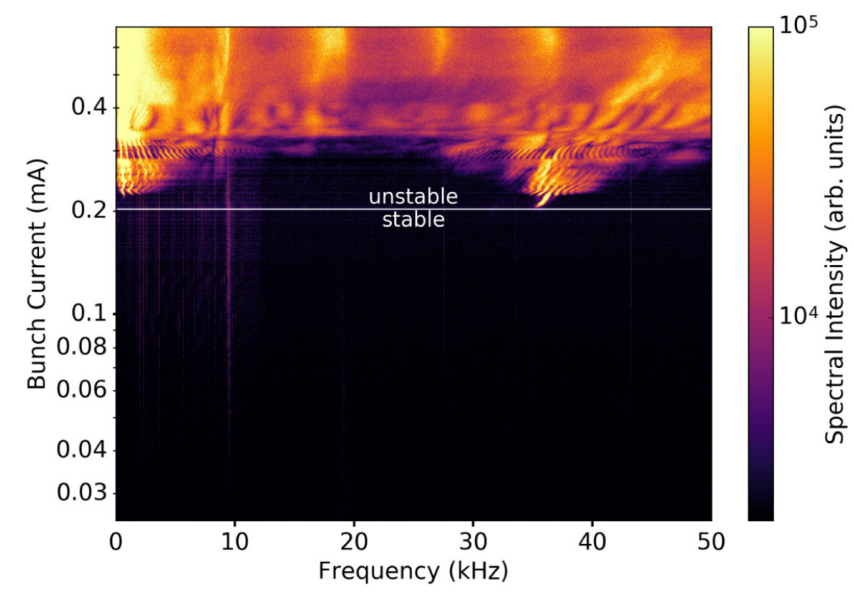

FIG. 1. Spectrogram of the fluctuations of the THz intensity as a function of the decaying bunch current, showing the microbunching instability. It was obtained in a measurement lasting several hours while the bunch current decreased. No shortbunch-length bursting occurs, as the bunch was not compressed strongly enough. The measurement was taken for settings resulting in a shielding of $\Pi \approx 1.41\left(f_{\mathrm{s}}=9.4 \mathrm{kHz}, \quad V_{\mathrm{rf}}=\right.$ $1048 \mathrm{kV}, \tau_{d}=10.6 \mathrm{~ms}$ ). reduction of the measurement time down to one second [12]. This so-called snapshot measurement technique was used for measuring the bunch-current dependence of the behavior as well as the threshold of the instability.

\section{B. Short-bunch-length bursting}

For most machine settings, the beam is stable for all currents below the bursting threshold (see Fig. 1). Nevertheless, observations show that at KARA, for a momentum compaction factor $\alpha_{\mathrm{c}} \leq 2.64 \times 10^{-4}$ combined with high rf voltages $\left(V_{\mathrm{rf}}>1100 \mathrm{kV}\right)$ leading to a natural bunch length $\sigma_{\mathrm{z}, 0} \leq 0.723 \mathrm{~mm} \triangleq 2.43 \mathrm{ps}$, an instability occurs again for bunch currents below the main bursting threshold; see Fig. 2. This spectrogram was obtained by a snapshot measurement within one second. To compensate for the limited current resolution of this measurement method, the filling pattern was chosen in such a way that the region of interest with small bunch currents is sampled with a sufficient resolution (see Supplemental Material [19]). This is visible in the limited bunch-current resolution in the upper part in Fig. 2. The spectrogram shows the lower bound of the main bursting and the complete occurrence of the short-bunch-length bursting. This second region of instability occurred at bunch currents between 0.038 and $0.016 \mathrm{~mA}$ for the measured machine settings.

The frequencies of the intensity fluctuations (in Fig. 2) are, for currents above the main bursting threshold, slightly above twice the synchrotron frequency $\left(2 \times f_{\mathrm{s}}=2 \times\right.$ $6.55 \mathrm{kHz}$ ). For the SBB, they are slightly below twice the synchrotron frequency and approach this frequency with a decreasing bunch current. A second frequency line at the first harmonic of the intensity fluctuation is visible (below $4 \times f_{\mathrm{s}}$ ). (See Supplemental Material for $\mathrm{THz}$

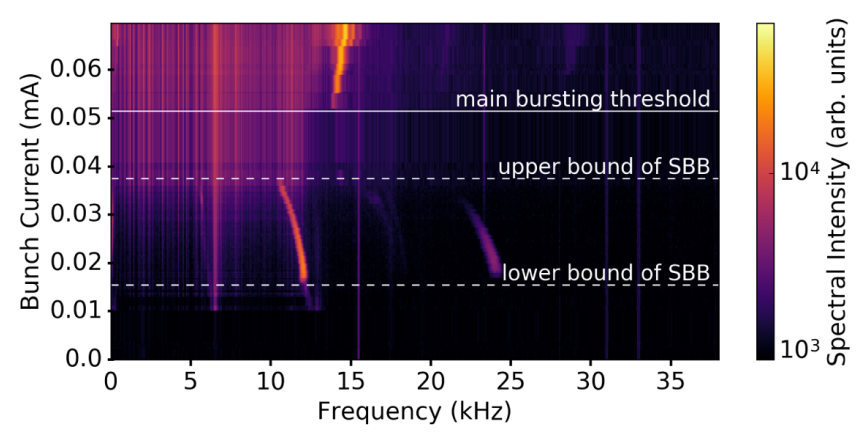

FIG. 2. Snapshot spectrogram of the fluctuations of the $\mathrm{THz}$ intensity as a function of the bunch current for a synchrotron frequency of $6.55 \mathrm{kHz}\left(\Pi \approx 0.74, V_{\mathrm{rf}}=1400 \mathrm{kV}, \tau_{d}=10.6 \mathrm{~ms}\right)$. Below the end of the microbunching instability (main bursting threshold) around $0.052 \mathrm{~mA}$, a second unstable region is clearly visible between 0.038 and $0.016 \mathrm{~mA}$. The current bins were chosen such that a high bunch-current resolution in the region of the short-bunch-length bursting was achieved. For this measurement, approximately 115 bunches were filled. 
signals as a function of turns at different bunch currents [20].)

\section{Results}

Snapshot measurements of the lower current range, similar to Fig. 2, were taken for different values of the momentum compaction factor and the natural bunch length by changing the magnet optics as well as the rf voltage. The scanned parameter range reached for $\alpha$ from $9.94 \times 10^{-3}$ down to $1.51 \times 10^{-3}$ and for $V_{\text {rf }}$ from 524 up to $1500 \mathrm{kV}$.

The bunch currents at the lower and upper bounds of the short-bunch-length bursting as well as the main bursting threshold for each measurement are displayed in Fig. 3 using the dimensionless parameters $S_{\mathrm{CSR}}$ and $\Pi$ [Eqs. (2) and (3)] following the notation of Ref. [3].

Bursting thresholds measured at machine settings, where no short-bunch-length bursting occurs (for more details, see [12]), show that the main bursting threshold is described by Eq. (1) and is independent of the occurrence of shortbunch-length bursting.

The highest value of the shielding parameter $\Pi$ where the short-bunch-length bursting occurs at KARA (rightmost red square in Fig. 3) is $\Pi_{\text {highest } S B B}=0.845 \pm 0.013$. The smallest value of the shielding parameter where the shortbunch-length bursting does not occur (leftmost green diamond in Fig. 3) is at $\Pi_{\text {no SBB }}=0.835 \pm 0.017$ and therefore smaller than $\Pi_{\text {highest SBB }}$. This small difference is expected and caused by the fact that the two values $\left(\Pi_{\text {no SBB }}\right.$ and $\left.\Pi_{\text {highest SBB }}\right)$ were obtained for different combinations of the momentum compaction factor and $\mathrm{rf}$ voltage with similar values of $\Pi$, however, different values for $\beta\left(\beta_{\mathrm{noSBB}}=2.59 \times 10^{-3}\right.$ and $\left.\beta_{\text {highest SBB }}=1.88 \times 10^{-3}\right)$.

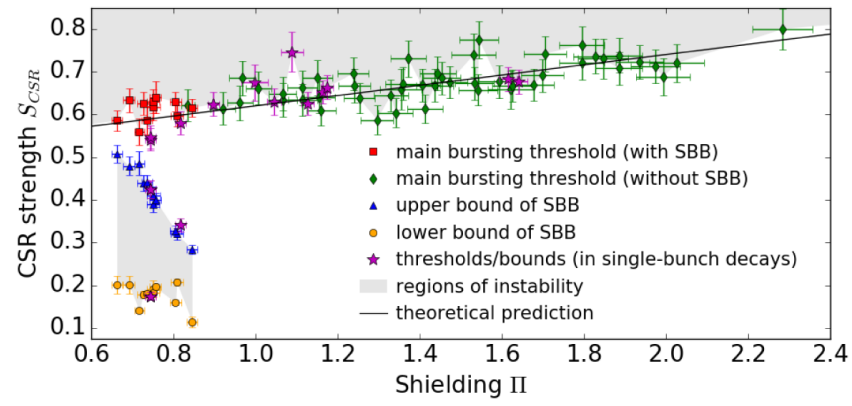

FIG. 3. CSR strength vs shielding of thresholds from snapshot measurements at different settings of the machine parameters compared to the linear scaling law given by Eq. (1) (line). The lower bound (orange disks) as well as the upper bound (blue triangles) of the SBB are shown. The main bursting threshold is shown in red (squares) for machine settings where short-bunchlength bursting occurred and in green (diamonds) for settings where it did not occur. The purple stars represent thresholds and bounds which were obtained from a full decay of a single bunch and not from snapshot measurements. The error bars display the one standard deviation uncertainties calculated from the measurement errors.
As described in Ref. [21], the range of $\Pi$ where the weak instability occurs is the bigger the smaller $\beta$ is.

The overall limit agrees within uncertainties with the results obtained by Bane, Cai, and Stupakov using their VFP solver [3]. There, the authors observed a dip around $\Pi=0.7$, while the threshold for $\Pi=1$ is again on the theoretical calculated linear scaling law given by Eq. (1). Values below $\Pi=0.66$ were not accessible for our measurements, which precludes the possibility to check if the short-bunch-length bursting vanishes for even smaller values of the shielding parameter, as predicted by calculations in Ref. [3].

\section{SIMULATIONS}

The upper and lower limits in the bunch current of the short-bunch-length bursting are expected to depend not only on the natural bunch length $\sigma_{z, 0}$ but also on $\beta=1 /\left(2 \pi f_{\mathrm{s}} \tau_{\mathrm{d}}\right)$, which relates the longitudinal damping time $\tau_{\mathrm{d}}$ and the synchrotron frequency $f_{\mathrm{s}}$. For the measurements presented here, the synchrotron frequency changes due to the different values of the rf voltage and the momentum compaction factor, while the damping time stays constant. This means that $\beta$ is different for the different measurement points ranging in the presented measurements from $\beta=1.13 \times 10^{-3}$ to $\beta=3.33 \times 10^{-3}$. As the simulations in Ref. [3] were carried out only for the fixed value $\beta=1.25 \times 10^{-3}$, new simulations were done for each measurement point using exactly the parameters of the respective measurement. The VFP solver used for these additional simulations is presented in Ref. [22], and a comparison between the simulation results and measurement done at MLS and BESSY (Berliner Elektronenspeicherring-Gesellschaft für Synchrotronstrahlung m.b.H.) is given in [13].

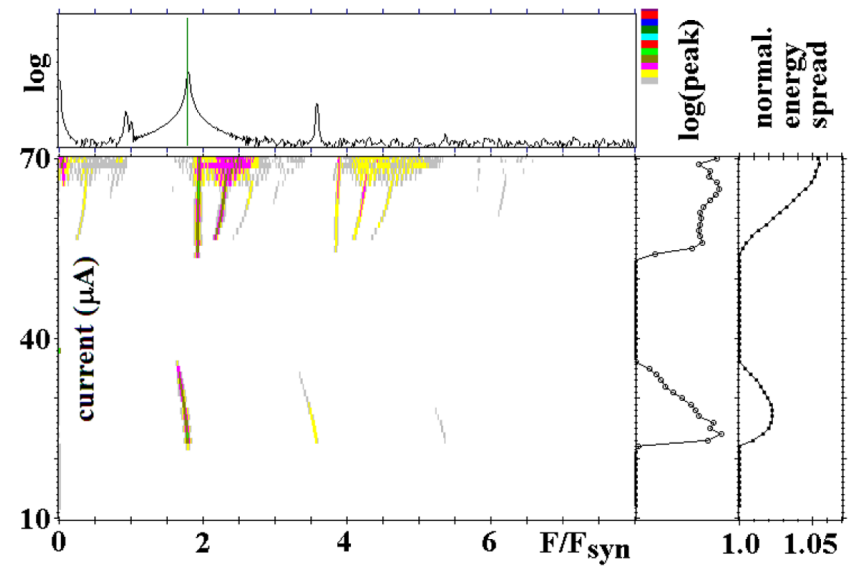

FIG. 4. Simulated spectrogram showing the end of the microbunching instability (main bursting threshold) around $54 \mu \mathrm{A}$ as well as the short-bunch-length bursting between 36 and $22 \mu \mathrm{A}$. The frequencies are directly below 2 and 4 times the synchrotron frequency, similar to the frequencies observed in the corresponding measurements (see Fig. 2) [21]. 


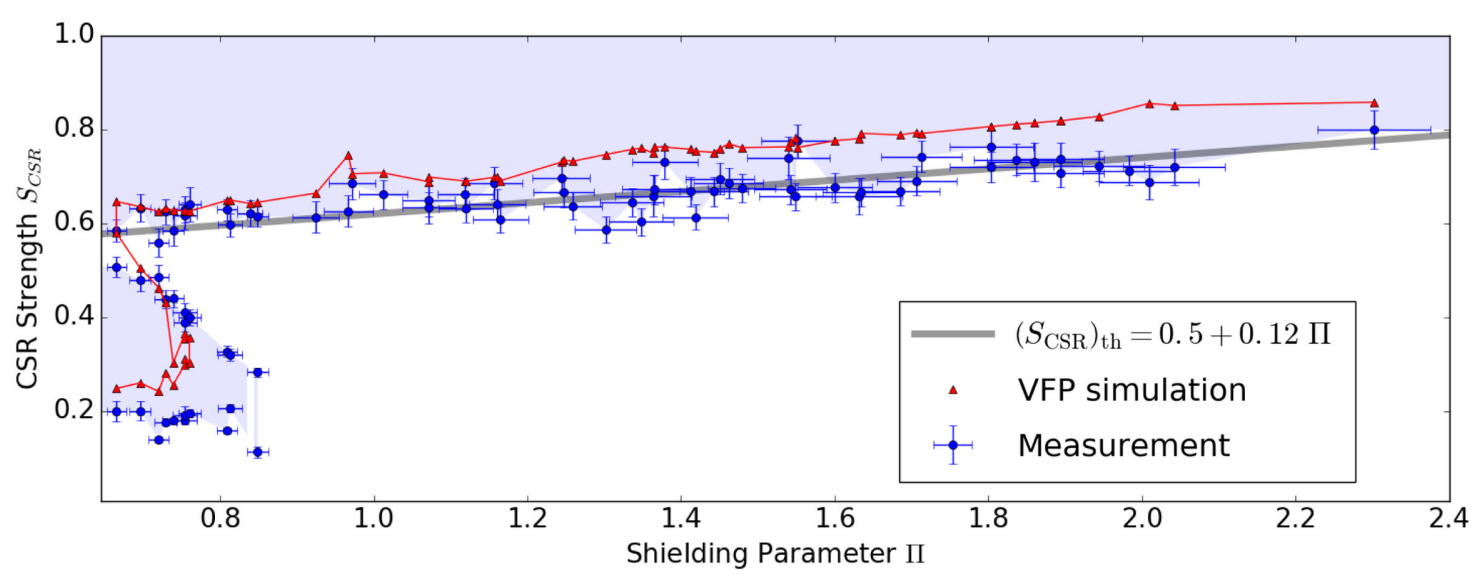

FIG. 5. CSR strength at the bursting thresholds as a function of the shielding parameter for measurements and VFP solver calculations for different machine settings. The measured area of instability is indicated as a light blue area and confined by the measured thresholds (blue disks, already shown in Fig. 3) with the error bars displaying the standard deviation error of each measurement. The red triangles show the results from the VFP solver calculations at the corresponding machine settings (red line to guide the eye). The gray line indicates the linear scaling law for the main bursting threshold given by Eq. (3).

Figure 4 shows a spectrogram calculated from the simulated phase space [21]. Similar to the measurements, a second region of instability corresponding to the shortbunch-length bursting is visible between 20 and $41 \mu \mathrm{A}$, well below the main bursting threshold at $54 \mu \mathrm{A}$. The dominant frequencies above the main threshold as well as in this second instability region are close to 2 and 4 times the synchrotron frequency, showing the same structure as the corresponding measurement (Fig. 2), indicating similar dynamics (of the fluctuations in the longitudinal particle distribution) close to the main threshold and in the SBB region.

The simulation also reveals that, in the stable area between the short-bunch-length bursting and the main bursting threshold, the energy spread equals the natural energy spread and is not increased as it is during the instability (see Fig. 4), confirming the first simulations done in Ref. [3].

The simulations yield thresholds which are higher by about $10 \%$ in comparison to the linear scaling law [3] as discussed above (see Sec. II). The overall behavior is in good agreement with the measurements. The CSR strengths at the thresholds obtained from the VFP solver are shown in Fig. 5 (red triangles) as a function of the shielding parameter.

\section{COMPARISON}

Figure 5 shows again clearly that, in the measurement as well as in the new VFP calculations, a range of $\Pi$ exists where unstable $\mathrm{THz}$ emission also occurs below the threshold given by the simple linear scaling law [Eq. (3)], as already shown by Ref. [3]. Our measurements as well as simulations show a stable area between the two regions of instability.
The range in $\Pi$ as well as in $S_{\mathrm{CSR}}$ where the short-bunchlength bursting occurs depends on $\beta$. For the parameters in our measurements, the simulations give an upper limit for the occurrence of short-bunch-length bursting of $\Pi=0.76$, while the measurements show short-bunch-length bursting up to $\Pi_{\text {highest SBB }}=0.845 \pm 0.013$, resulting in a small range of $\Pi$ where short-bunch-length bursting is observed by measurements and not in the simulations. Also, the lower bound of the short-bunch-length bursting in the CSR strength differs slightly between the calculations and the measurements. The measurements show instability at an even lower CSR strength (corresponding to a lower bunch current) than the calculations. This could be related to the fact that, in general, the threshold values obtained by the simulation are systematically slightly higher than the ones measured. The average difference is $7 \mu \mathrm{A}$ for the main threshold current ranging from 40 to $400 \mu \mathrm{A}$.

Lower values for the thresholds in the measurements cannot be explained by too-insensitive $\mathrm{THz}$ detectors, as this would result in an overestimation of the measured thresholds. Also, systematic influences on the measured thresholds due to multibunch effects in the used snapshot measurements can be excluded, as thresholds measured in pure single-bunch decays agree with the ones from snapshot measurements (see Fig. 3).

A small discrepancy consistent with the one observed could be caused due to our measurement method. For small fluctuations of the machine settings, the measurements would give the absolute floor of the corresponding thresholds, while the simulation gives a value corresponding more to the average threshold for the machine settings, leading to a small discrepancy. Such fluctuations in the machine could occur in the rf voltage and the current in the magnets and, thus, the magnet optics and the momentum compaction factor. 
Another potential source for deviations could come from assumptions used in the simulation. For example, the longitudinal damping time, an essential component in the solution of the VFP equation, was obtained by beam dynamics calculations which did not include CSR. Furthermore, as described above, the VFP calculations consider only the simple parallel plates model. The small discrepancy between the measured thresholds and the calculated ones could indicate additional impedance contributions. For example, considering an additional geometric impedance for an aperture like a scraper leads to a slightly lower simulated threshold [23]. Also, the impedance of edge radiation is mainly resistive and would lead, if considered in the simulation, to a lower threshold. Last but not least, a stronger CSR interaction than expected from the simple circular orbit simulated could be caused by an interaction extending into the straights behind the dipoles.

\section{SUMMARY}

The short-bunch-length bursting observed at KARA for certain machine settings corresponds to the behavior observed in the results of the Vlasov-Fokker-Planck solver calculations first published by Bane, Cai, and Stupakov [3]. This second region of instability below the bursting threshold of the main microbunching instability occurred in the measurements for values of the shielding parameter smaller than $\Pi=0.85$. As the occurrence of short-bunch-length bursting depends not only on the natural bunch length but also on the ratio of the synchrotron frequency and the longitudinal damping time, new VFP solver calculations were performed at the exact conditions of the measurements. This simulation result shows slightly higher values for the thresholds with an average difference of $7 \mu \mathrm{A}$, but the overall behavior is in good agreement with the measurements. The small deviations might be caused by the floor in the determination of the thresholds from the measurements, which would lead to an underestimation in the presence of small parameter fluctuations of the machine settings. Another possible explanation is an additional impedance contribution or a stronger CSR interaction that is not covered by the simple model simulated. The latter could be caused by the interaction of the bunches with their CSR extending into the straights behind the dipoles.

\section{ACKNOWLEDGMENTS}

We thank Karl Bane for his questions concerning the presence of this second region of instability at KARA. We thank the infrared group at KARA and, in particular, M. Süpfle for their support during the beam times at the Infrared 2 beam line. Furthermore, we thank the THz group for inspiring discussions. This work has been supported by the German Federal Ministry of Education and Research (Grant No. 05K13VKA), the Helmholtz Association (Contract No. VH-NG-320), and the Helmholtz International Research School for Teratronics (HIRST). E. Blomley and J. G. acknowledge the support by the DFG-funded Doctoral School "Karlsruhe School of Elementary and Astroparticle Physics: Science and Technology.” M. B., J. L. S., and P. Schönfeldt acknowledge the support of the Helmholtz International Research School for Teratronics (HIRST).

[1] G. Stupakov and S. Heifets, Beam instability and microbunching due to coherent synchrotron radiation, Phys. Rev. ST Accel. Beams 5, 054402 (2002).

[2] J. M. Byrd, W. P. Leemans, A. Loftsdottir, B. Marcelis, M. C. Martin, W. R. McKinney, F. Sannibale, T. Scarvie, and C. Steier, Observation of Broadband Self-Amplified Spontaneous Coherent Terahertz Synchrotron Radiation in a Storage Ring, Phys. Rev. Lett. 89, 224801 (2002).

[3] K. L. F. Bane, Y. Cai, and G. Stupakov, Threshold studies of the microwave instability in electron storage rings, Phys. Rev. ST Accel. Beams 13, 104402 (2010).

[4] A.-S. Müller, B. Balzer, C. Caselle, N. Hiller, M. Hofherr, K. Ilin, V. Judin, B. Kehrer, S. Marsching, S. Naknaimueang, M. Nasse, J. Raasch, A. Scheuring, M. Schuh, M. Schwarz, M. Siegel, N. Smale, J. Steinmann, P. Thoma, M. Weber, and S. Wuensch, Studies of bunch-bunch interactions in the ANKA storage ring with coherent synchrotron radiation using an ultra-fast terahertz detection system, in Proceedings of the 4th International Particle Accelerator Conference, IPAC-2013, Shanghai, China, 2013 (JACoW, Shanghai, China, 2013), p. 109.

[5] M. Brosi, E. Blomley, E. Bründermann, N. Hiller, B. Kehrer, A.-S. Müller, M. Schedler, M. Schuh, P. Schönfeldt, and J.L. Steinmann, Systematic studies of short bunch-length bursting at ANKA, in Proceedings of the International Particle Accelerator Conference (IPAC'16), Busan, Korea, 2016, International Particle Accelerator Conference No. 7 (JACoW, Geneva, Switzerland, 2016), pp. 1662-1665.

[6] M. Ries, J. Feikes, P. Schmid, G. Wüstefeld, and A. Hoehl, THz bursting thresholds measured at the metrology light source, in Proceedings of the 3rd International Particle Accelerator Conference, New Orleans, LA, 2012 (IEEE, Piscataway, NJ, 2012), p. 3030.

[7] W. Shields, R. Bartolini, G. Boorman, P. Karataev, A. Lyapin, J. Puntree, and G. Rehm, Microbunch instability observations from a $\mathrm{THz}$ detector at diamond light source, J. Phys. Conf. Ser. 357, 012037 (2012).

[8] T. Agoh, Steady fields of coherent synchrotron radiation in a rectangular pipe, Phys. Rev. ST Accel. Beams 12, 094402 (2009).

[9] Y. Cai, Scaling law of coherent synchrotron radiation in a rectangular chamber, Phys. Rev. ST Accel. Beams 17, 020702 (2014).

[10] H. Wiedemann, Synchrotron radiation in storage rings, Handbook of Accelerator Physics and Engineering, 2nd ed. (World Scientific, Singapore, 2013), Chap. 3.1.4, pp. 220-223.

[11] R. L. Warnock and J. A. Ellison, A General method for propagation of the phase space distribution, with application 
to the sawtooth instability, in The physics of high brightness beams. Proceedings of the 2nd ICFA Advanced Accelerator Workshop, Los Angeles, USA, 1999 (SLAC National Accelerator Laboratory, Menlo Park, CA, 2000), pp. 322-348, http://www-public.slac.stanford.edu/sciDoc/docMeta.aspx? slacPubNumber=SLAC-PUB-8404.

[12] M. Brosi, J. L. Steinmann, E. Blomley, E. Bründermann, M. Caselle, N. Hiller, B. Kehrer, Y.-L. Mathis, M. J. Nasse, L. Rota, M. Schedler, P. Schönfeldt, M. Schuh, M. Schwarz, M. Weber, and A.-S. Müller, Fast mapping of terahertz bursting thresholds and characteristics at synchrotron light sources, Phys. Rev. Accel. Beams 19, 110701 (2016).

[13] P. Kuske, CSR-driven longitudinal single bunch instability thresholds, in Proceedings of the 4th International Particle Accelerator Conference, IPAC-2013, Shanghai, China, 2013 (JACoW, Shanghai, China, 2013), WEOAB102.

[14] Advanced Compound Semiconductor Technologies (ACST) $\mathrm{GmbH}$, UWB Quasi-optical Detectors up to $2 \mathrm{THz}$, flyer.

[15] Y.-L. Mathis, B. Gasharova, and D. Moss, Terahertz radiation at ANKA, the new synchrotron light source in karlsruhe, J. Biol. Phys. 29, 313 (2003).

[16] M. Caselle, M. Brosi, S. Chilingaryan, T. Dritschler, E. Hertle, V. Judin, A. Kopmann, A.-S. Müller, J. Raasch, M. Schleicher, M. Siegel, N. Smale, J. Steinmann, M. Vogelgesang, M. Weber, and S. Wuensch, Commissioning of an ultra-fast data acquisition system for coherent synchrotron radiation detection, in Proceedings of the 2014 International Particle Accelerator Conference (2014), p. 3497, http://accelconf.web.cern.ch/AccelConf/ IBIC2014/papers/moczb1.pdf.

[17] M. Caselle, M. Brosi, S. Chilingaryan, T. Dritschler, N. Hiller, V. Judin, A. Kopmann, A.-S. Müller, L. Petzold,
J. Raasch, L. Rota, M. Siegel, N. Smale, J. Steinmann, M. Vogelgesang, M. Weber, and S. Wuensch, A picosecond sampling electronic 'KAPTURE' for terahertz synchrotron radiation, in Proceedings of IBIC'14 Monterey, CA, USA (2014).

[18] E. Hertle, E. Huttel, A.-S. Müller, N. Smale, D. Teytelman, and M. Höner, First Results of the new bunch-by-bunch feedback system at ANKA, in Proceedings of 5th International Particle Accelerator Conference IPAC'14 (2014), p. 1739.

[19] See Supplemental Material at http://link.aps.org/ supplemental/10.1103/PhysRevAccelBeams.22.020701 for a plot of the filling pattern used during the snapshot measurements.

[20] See Supplemental Material at http://link.aps.org/ supplemental/10.1103/PhysRevAccelBeams.22.020701 for a plot of THz signals at different bunch currents in the main bursting region and the SBB region.

[21] P. Kuske, Short bunches at the transition from strong to weak longitudinal instability, in Proceedings of the International Particle Accelerator Conference (IPAC'17), Copenhagen, Denmark, 2017, International Particle Accelerator Conference No. 8 (JACoW, Geneva, Switzerland, 2017), pp. 3696-3699.

[22] P. Kuske, Calculation of longitudinal instability threshold currents for single bunches, in Proceedings of ICAP2012, Rostock-Warnemünde, Germany (2012), THSDC3, accelconf.web.cern.ch/AccelConf/ICAP2012/papers/thsdc3 .pdf.

[23] P. Schönfeldt, Simulation and measurement of the dynamics of ultra-short electron bunch profiles for the generation of coherent $\mathrm{THz}$ radiation, Ph.D. thesis, Karlsruhe Institute of Technology, 2018. 